\title{
Evaluation of SIP Signalling and QoS for VoIP Over OLSR MANET Routing Protocol
}

\author{
Mazin Alshamrani, Haitham Cruickshank, Zhili Sun, Basil Elmasri, and Vahid Fami, \\ Centre for Communication System Research (CCSR), \\ University of Surrey, \\ Guildford, Surrey, UK \\ m.alshamrani@surrey.ac.uk, h.cruickshank@surrey.ac.uk,z.sun@surrey.ac.uk, \\ b.elmasri@surrey.ac.uk, v.fami@surrey.ac.uk
}

\begin{abstract}
This paper evaluates the SIP based VoIP applications over the Optimized Link State Routing protocol (OLSR) as a proactive routing protocol for Mobile Ad Hoc Networks (MANET) using Static, Uniform, and Random mobility models. The evaluation considered PCM, LQS, IPTelephony, and GSM voice codecs to study the SIP signaling performance and the voice Quality of Service $(\mathrm{QoS})$ for VoIP calls over OLSR MANET. The simulation efforts performed in OPNET Modeler 17.1. The results show that VoIP over OLSR MANET has good performance over Static and Uniform mobility models while it has variable performance with Random models. SIP signaling has large delays compared with the voice signaling which reduce the VoIP performance and increases the call's duration. In addition, GSM and LQS based VoIP calls have an acceptable level of QoS while PCM and IPTelephony based VoIP calls have a low level of QoS over different types of mobility models. Furthermore, the location and the mobility of SIP server affect the number of hops and the SIP signaling performance between the different parties of the VoIP call.
\end{abstract}

Keywords- VoIP; SIP; QoS; PCM; IP-Telephony; LQS; GSM; MANET; OLSR; OPNET

\section{INTRODUCTION}

MANET is one of the most common wireless networks with dynamic distributions of mobile devices that move in different types of mobility systems [1]. MANET has different types of routing protocols, and each routing protocol has its own characteristics over different types of applications and mobility models. This evaluation study considered OLSR as a proactive routing protocol for MANET. On the other hand, voice over IP (VoIP) is one of the most common applications over different types of network systems. Different factors affect the VoIP QoS over OLSR MANET such as the mobility model, voice codec, physical distance between calls parties, hops number, node capacity, WLAN technology system, and calls durations. The main aim of the evaluation study is to examine the QoS and SIP signaling for VoIP applications over OLSR MANET with moderate node capacity, different types of mobility models, and different voice codecs.

In this evaluation study, the IEEE $802.11 \mathrm{n}$ considered as the WLAN technology which represents the physical layer technology for the implemented MANET. In addition, we considered IPv4 as the addressing mode for all MANET nodes. IPv4 is still widely used for different network systems. The simulation works are implemented using OPNET modeler. OPNET Modeler is a commercial network simulation environment for network modelling and simulation. It allows users to design and study communication networks, devices, protocols, and applications with flexibility and scalability. It simulates the network graphically and gives the graphical structure of actual networks and network components. The SIP based VoIP applications consist of two types of signaling: SIP signaling (TCP based) and voice Signaling (UDP based). Both signaling systems have an impact on the VoIP QoS.

\section{A. SIP Signalling:}

SIP is a signaling protocol defined by SIP Working Group, within Internet Engineering Task Force (IETF). The protocol was published as IETF (RFC 2543) and currently has the status of a proposed standard [2]. SIP is commonly used for controlling the multimedia communication sessions such as voice and video calls over Internet Protocol (IP). The SIP session can include one or more Participants/Applications and can be used for creating, modifying, and terminating two or more participant sessions by consisting of one or more media streams. SIP is a text encoded protocol with a built in code which allows different type of modifications and extensions. The modifications could be applied on the addresses, ports, participant invitations, and adding/deleting media streams [2]. SIP is an application layer protocol designed to be independent of the existing transport layer and it depends on the supported Internet protocols. Moreover, SIP can run on the top of the TCP/IP, UDP/IP, or Stream Control Transmission Protocol (SCTP) [3]. These essential characteristics allow SIP to provide many valuable features and services for different types of network systems such as call control services, mobility, interoperability with exist telephone systems, and more.

The SIP based VoIP calls depend on three main stages: the registration stage, the Call initiation stage, and the call termination stage. These stages are depending on the SIP Proxy Server to relay the connectivity between different callers. The delays of the SIP signals in all stages affect the performance of VoIP calls. As SIP is a TCP based application layer signaling system, all the TCP timers (retransmission, and Round Trip Timer (RTT) are important factors for the overall structure of the SIP connectivity 
system. The average acceptable delays in SIP signaling system are between 0.150 to 0.350 Seconds [3].

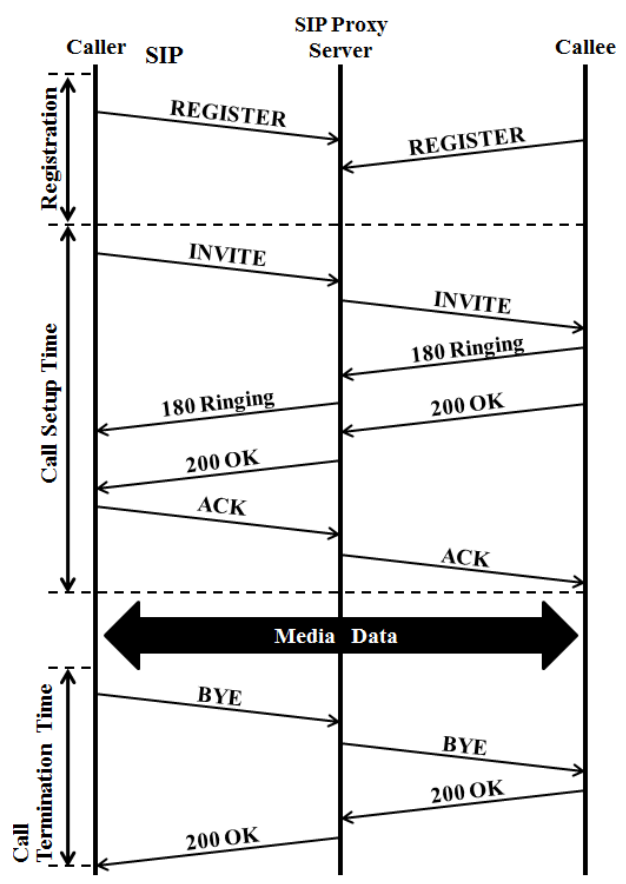

Figure 1. SIP Signalling Flow for SIP Based VoIP Applications.

\section{B. Voice Signalling:}

Voice traffic transferred over a special protocol system known as the Real Time Protocol (RTP) which is based on UDP. For SIP based VoIP applications, the call initiation stage which activates the media data transfer process directly from the Caller to the Callee using one of the supported voice codecs. This research study used to apply four types of voice codecs: Pulse Code Modulation (PCM), Low Quality Speech (LQS), IP Telephony, and GSM FR Quality Speech [3]. Each voice codec has its own characteristics and voice quality which affected by the number of hops, routing protocol, voice load, background noise, node movement, mobility models, bandwidth, traffic congestions and etc. The QoS parameters Voice applications are Jitter, End-To-End Delay, Amount of Traffic Received, and the amount of Traffic Sent/Received. The voice coding exists in the sender side, and the voice decoding exists in the receiver side. Both sides have its own coding delays depends on the voice compression performance in the callers' devices.

\section{MANET Overview:}

In telecommunication systems, MANET is as selfconfigured unlike nodes, creating infrastructure-less network, connected with different Wireless Networks as nodes which exchange data packets without a central control system [1]. There are three types of routing protocols in MANET:
- Reactive routing protocols: On-demand protocols that discover the routes when needed. It considered as source-initiated route discovery protocols (e.g. Dynamic Source Routing (DSR), Ad hoc On Demand Distance Vector (AODV), and Temporally Ordered Routing Algorithm (TORA)).

- Proactive routing protocols: Traditional distributed protocols that use the shortest paths based on periodic updates, however, they have high routing overhead (e.g. OLSR, and Destination Sequenced Distance Vector (DSDV)).

- Hybrid routing protocols: Combined functionality of the reactive and the proactive routing protocols with hybrid routing capabilities (e.g. Zone Routing Protocol (ZRP)).

\section{OLSR Overview:}

OLSR [1] is a proactive routing protocol with a table driven protocol system that stores and updates the network routes. Whenever a route is needed, OLSR offers the route immediately without any initial delays of route exploration. In OLSR, some candidate nodes called multipoint relays (MPRs) are selected to have the responsibility of forwarding the broadcast packets during traffic flooding process. This technique reduces the overhead of packet transmission compared with the flooding mechanism [4]. OLSR performs a hop by hop routing system, where each node uses its most updated routing information to route and deliver the packets. Each MPR covers all nodes that are two hops away. A node selects it's MPRs with control messages called (HELLO) messages. The (HELLO) messages are used to ensure bidirectional links with neighbors, and it sent at certain time intervals. The Nodes broadcast Topology Control (TC) messages used to determine its own MPRs [4].

\section{RELATED WORKS}

Limited number of researches worked with the evaluation of real-time applications over the proactive routing protocols in general and OLSR in particular. The OLSR performance used to be evaluated with other types of routing protocols. Most of the evaluation works considered the File Transfer Protocol (FTP) or Constant Bit Rate (CBR) traffic with a different number of MANET nodes. In [4], an evaluation study used to compare different performance parameters between the most popular routing protocols in MANET: OLSR (proactive), TORA (reactive), and Geographic Routing Protocol (GRP) (hybrid). The study exploited FTP traffic over models with different node capacity. The results show that OLSR has the best performance in terms of load and throughput. However, it has considerable delays and routing overhead compared with other routing protocols as a result of the MPR nodes selection. In [5], a comparison between OLSR and TORA in terms of delay, retransmissions, and data drop, has shown that OLSR behaves with nodes update to reduce the delays and increase the throughput while the retransmission attempts considered as a real problem in OLSR. 
A method of proactive MANET routing protocol evaluation applied to the OLSR protocol as proposed to [6]. It analyzed the performance of the OLSR in a medium sized MANET clusters using data from the MANIAC Challenge project specifically OLSR evaluation. A performance evaluation study about AODV and OLSR routing protocols under a realistic radio channel characteristics used to be implemented in NS-2 with Nakagami fading model in [7]. The study used CBR traffic with a uniform mobility model for $40 \mathrm{kph}$ speed. The results show that under realistic channel conditions in both routing protocols failed to deliver a good number of data packets to the destination nodes in highly fading environments. In [8], a study used to compared DSR, OLSR and ZRP using different mobile scenarios generated by Random Mobility model for MANET using CBR (UDP) traffic. The study used NS-2 and shows that OLSR has low average Jitter and End-to-End delay while it has high throughput. [9] Discussed the impact of the mobility models and the density of nodes on the performances of OLSR by using the real-time VBR (MPEG4) and the Constant Bit Rate (CBR) traffic. The paper compared the performance on both cases in ns-2 over three mobility models: Random Waypoint, Random Direction, and Mobgen Steady State. The simulation results show that the OLSR behavior changes according to the used traffic and the mobility model where Random Waypoint has the optimal throughput.

A proposed QoS extension model for OLSR MANET presented and evaluated in OPNET for voice applications [10]. The simulation result shows an improvement of the packets delivery ratio by using the proposed QoS support model for voice communication over MANET compared with native OLSR. The study focused on the voice signaling using PCM voice codec. In [11], a study illustrated the performance of real-time streaming media over a mesh OLSR based network systems. It examined the effect of mobility and background traffic on carried load and jitter for media applications using IEEE 802.11 MAC/PHY with EMANE software emulator.

\section{Simulation SETUP PARAMETERS}

In this evaluation study, we considered the IEEE 802.11n as the wireless networking the standard for OLSR based MANET. The simulation works applied over four types of mobility models: Static, Uniform, Random, and Random All. Table 1 represents a brief summary of the simulation parameters. These parameters identified depending on the features and the capabilities of OLSR MANET and VoIP applications compared with other evaluation studies as in [4], [5] and [6]. Fig. 2 shows the design implementation of OLSR based MANET in OPNET regarding the identified simulation parameters in Table 1. This design used in all of the mobility models in this study. In the Static model, MANET's nodes are stable and not moving. In the Uniform model, all nodes are moving in the same direction with different speed ranges including the SIP server. In Random model, nodes are moving in different directions, but the SIP server is stable in the center of the simulation area. Fig. 3 shows the simulation implementation in OPNET for the
MANET Random mobility model after 20 seconds from the beginning of the simulation where each node, except the SIP Server, has its own mobility direction and speed depending on the identified random functionality of the node parameters. Finally, in Random All model, all nodes are moving in different directions, including the SIP server. The reason for examining Random mobility using two different models is to study and evaluate the effect of the SIP server mobility over the VoIP applications and the signaling QoS. All the assumptions and simulation works in this study are based on IPv4 MANET.

TABLE I. SIMULATION PARAMETERS IN OPNET

\begin{tabular}{|c|c|c|c|c|c|c|c|}
\hline \multirow{2}{*}{\multicolumn{2}{|c|}{$\begin{array}{c}\begin{array}{c}\text { Number of } \\
\text { Simulations }\end{array} \\
\text { Simulation } \\
\text { Duration: } \\
\end{array}$}} & & 128 \\
\hline & & \multicolumn{6}{|c|}{10 Minutes $=600$ Seconds } \\
\hline \multicolumn{2}{|c|}{$\begin{array}{l}\text { Mobility } \\
\text { Models: }\end{array}$} & \multicolumn{6}{|c|}{ Static, Uniform, Random, and Random All } \\
\hline \multicolumn{2}{|c|}{$\begin{array}{l}\text { Number of } \\
\text { nodes: }\end{array}$} & \multicolumn{2}{|c|}{25 nodes } & \multicolumn{2}{|c|}{$\begin{array}{c}\text { Area } \\
\text { Dimension: }\end{array}$} & \multicolumn{2}{|c|}{$1 \mathrm{~km} \mathrm{x} 1 \mathrm{~km}$} \\
\hline \multicolumn{2}{|c|}{$\begin{array}{l}\text { Node Speed } \\
\text { Range: }\end{array}$} & \multicolumn{6}{|c|}{$\begin{array}{c}\text { Uniform Speed between } 5.57 \mathrm{~m} / \mathrm{s}(20 \\
\mathrm{km} / \mathrm{hr}) \text { and } 12.5 \mathrm{~m} / \mathrm{s}(45 \mathrm{~km} / \mathrm{hr})\end{array}$} \\
\hline \multicolumn{2}{|c|}{$\begin{array}{l}\text { WLAN Physical } \\
\text { Characteristic: }\end{array}$} & \multicolumn{2}{|c|}{$802.11 \mathrm{n}$} & \multicolumn{2}{|c|}{ Data Rate: } & \multicolumn{2}{|c|}{$13 \mathrm{Mbps}$} \\
\hline \multicolumn{4}{|c|}{$\begin{array}{l}\text { Maximum Transmission } \\
\text { Range between Nodes: }\end{array}$} & \multicolumn{4}{|c|}{$\begin{array}{l}\text { from } 100 \text { meters to } 250 \\
\text { meters }\end{array}$} \\
\hline $\begin{array}{l}\text { Frequency } \\
\text { Band: }\end{array}$ & \multicolumn{3}{|c|}{$2.4 \mathrm{GHz}$} & \multicolumn{2}{|c|}{$\begin{array}{l}\text { Transmission } \\
\text { Power: }\end{array}$} & \multicolumn{2}{|c|}{$0.001 \mathrm{~W}$} \\
\hline $\begin{array}{l}\text { Packet } \\
\text { Size: }\end{array}$ & \multicolumn{3}{|c|}{512 Bytes } & \multicolumn{2}{|c|}{ Buffer Size: } & \multicolumn{2}{|c|}{32 Kbytes } \\
\hline \multicolumn{8}{|c|}{ B. Major OLSR Parameters and Values: Default } \\
\hline \multicolumn{3}{|c|}{$\begin{array}{c}\text { Hello Interval } \\
\text { (Seconds) }\end{array}$} & 2 & \multicolumn{3}{|c|}{$\begin{array}{l}\text { Neighbor Hold } \\
\text { Time (Seconds) }\end{array}$} & 6 \\
\hline \multicolumn{3}{|c|}{$\begin{array}{l}\text { Topology Control } \\
\text { (TC) Interval } \\
\text { (Seconds) }\end{array}$} & 5 & \multicolumn{3}{|c|}{$\begin{array}{l}\text { Topology Hold } \\
\text { Time (Seconds) }\end{array}$} & 15 \\
\hline \multicolumn{3}{|c|}{$\begin{array}{c}\text { Duplicate Message } \\
\text { Hold Time (Seconds) }\end{array}$} & 30 & \multicolumn{3}{|c|}{ Addressing Model } & IPv4 \\
\hline \multicolumn{8}{|c|}{ C. Applications: SIP Based VoIP } \\
\hline $\begin{array}{c}\text { SIP } \\
\text { Server } \\
\text { Connect } \\
\text { Timeout: }\end{array}$ & \multicolumn{2}{|c|}{$\begin{array}{c}\text { TCP } \\
\text { Based }\end{array}$} & \multicolumn{2}{|c|}{$\begin{array}{l}\text { Voice } \\
\text { Codec: }\end{array}$} & \multicolumn{3}{|c|}{\begin{tabular}{|l} 
PCM (G.711, $64 \mathrm{kbps})$ \\
LQS (G.723.1, 5.3 kbps) \\
IP-T (G.729 A, $8 \mathrm{Kbps})$ \\
GSM (GSM, 13 Kbps) \\
\end{tabular}} \\
\hline & Dura & tions & & er & Callee & $\begin{array}{l}\text { Nun } \\
\text { Calls } \\
\text { Sec }\end{array}$ & $\begin{array}{l}\text { ber of } \\
\text { in } 600 \\
\text { onds }\end{array}$ \\
\hline ( & 5 & & & 1 & Node 24 & & 15 \\
\hline & 10 & & No & 22 & Node 3 & & 50 \\
\hline & & & & e 5 & Node 20 & & 0 \\
\hline & & & & 15 & Node19 & & 5 \\
\hline $\begin{array}{c}\text { Maximum } \\
\text { Simultaneo }\end{array}$ & & Serv & & $\begin{array}{l}\text { Use } \\
\text { (Call }\end{array}$ & $\begin{array}{l}\text { Agent } \\
\text { r/Callee) }\end{array}$ & $\begin{array}{r}\text { Total } \\
\text { Calls } \\
\text { Sec }\end{array}$ & $\begin{array}{l}\text { VoIP } \\
\text { n } 600 \\
\text { nds } \\
\end{array}$ \\
\hline us Calls & & Sec & & $1 \mathrm{Ca}$ & Second & 220 & Calls \\
\hline
\end{tabular}




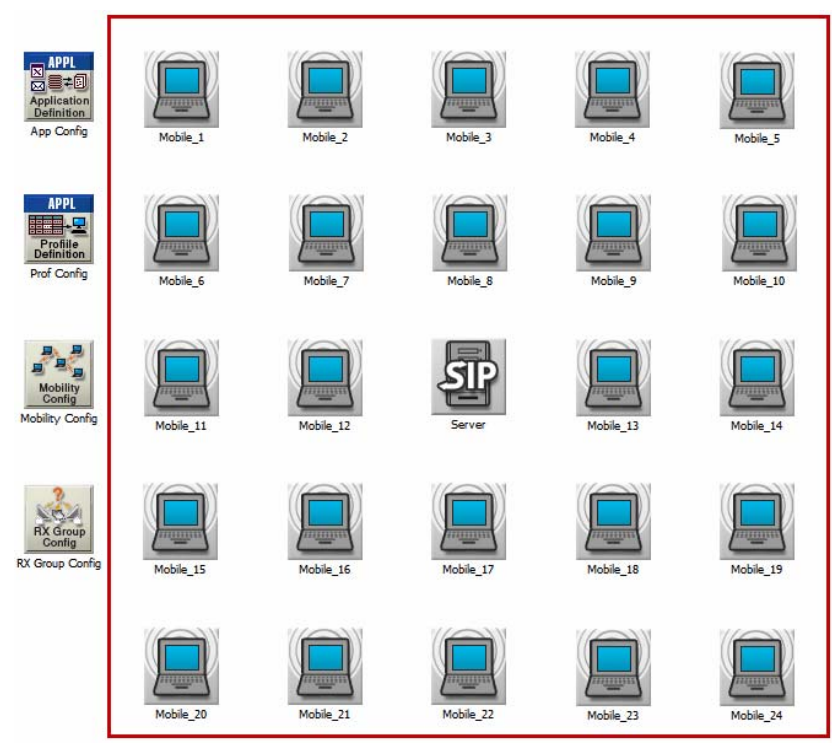

Figure 2. MANET Models implementation in OPNET. The red box represents the mobility area of $1 \mathrm{Km} \times 1 \mathrm{Km}$ for MANET nodes.

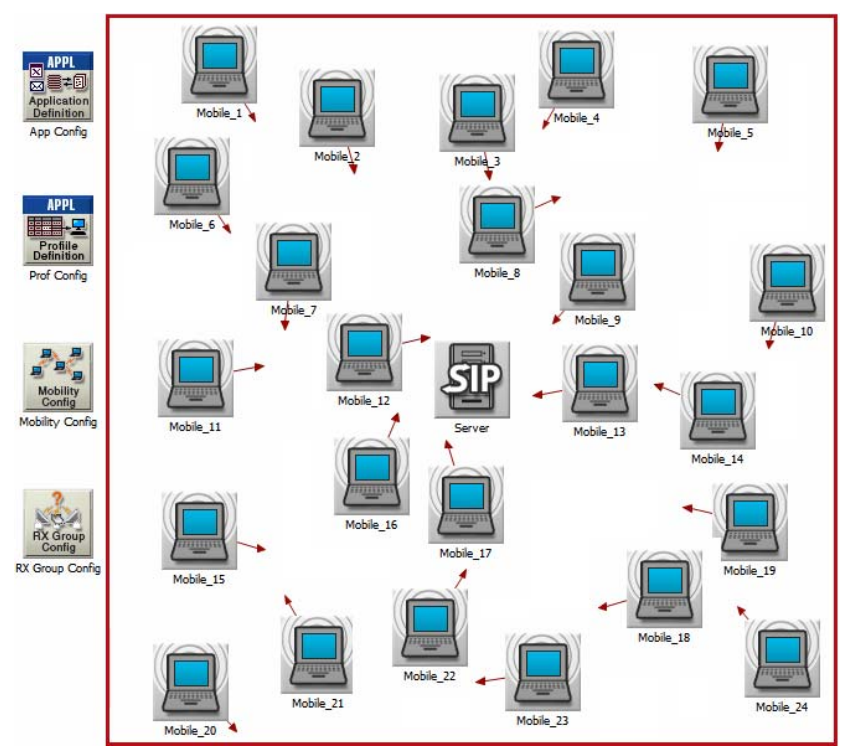

Figure 3. MANET Random Mobility Model at the simulation time: 20 Seconds. The red arrows represent the mobility direction for each node.

\section{RESULTS AND DiSCUSSION}

The simulation results focus on three main aspects for VoIP applications; the SIP signaling system between the call's parties, the voice QoS, and the related OLSR performance parameters. Each evaluation aspect is considered over the defined mobility models as explained in the previous section. The $\mathrm{X}$ axis in all results represents the simulation time in Seconds (S) which provides comparable graphical statistics between the mobility models and the voice codecs over the simulation time. The $\mathrm{Y}$ axis is variable from one figure to another. It represents the call's duration in
Seconds (S), the Number of Calls (N), or the Number of Bytes (B). In general, the performance of most of the VoIP applications has low stability at the beginning of the simulation. However, the performance of the VoIP calls in general reaches stability after 150 to 200 seconds from the start of the simulation and the results show comparable statistics for the VoIP related aspects. These delays happen at the beginning of the simulation and effects on the VoIP applications because of the initiation of the OLSR routing tables, and the registration process of the MANET nodes with SIP server.

Table 2 shows the total number and the percentage of successful and rejected VoIP calls based on the total number of generated VoIP calls over all completed simulation works. In general, all mobility models over different types of voice codecs have a percentage of delays and calls rejection. The best results are shown within the Uniform mobility model then the Static model while the worst results are in Random All model then the Random model. The mobility in the Uniform model causes some reductions in hop numbers between the call parties which is reflected in the performance and the number of achieved calls compared with the Static model. On the other hand, SIP server movements reduce the instantaneous connectivity for VoIP applications. The SIP server location has an effect on the application performance where the central position in the mobility area shown the best performance as the hops number between all nodes are kept to the minimum level. Furthermore, LQS and GSM voice codecs have the best percentage of successful VoIP calls over all mobility models while the PCM has the highest percentage of the rejected VoIP calls over all mobility models. This is because the LQS and the GSM have lower bit rates. Each call delay has an effect on next calls, as continues and unlimited calls are generated during the simulation time.

TABle II. Simulation Results, VoiP General Statistics

\begin{tabular}{|c|c|c|c|c|}
\hline $\begin{array}{l}\text { Mobility } \\
\text { Model }\end{array}$ & $\begin{array}{l}\text { Voice } \\
\text { Codec }\end{array}$ & $\begin{array}{l}\text { Total Successful } \\
\text { VoIP Calls out } \\
\text { of } \mathbf{2 2 0} \text { calls }\end{array}$ & $\begin{array}{c}\% \text { of } \\
\text { Successf } \\
\text { ul VoIP } \\
\text { calls }\end{array}$ & $\begin{array}{c}\text { \% of } \\
\text { Rejected } \\
\text { VoIP } \\
\text { calls }\end{array}$ \\
\hline \multirow{4}{*}{ Static } & PCM & 91 & $41.4 \%$ & $58.6 \%$ \\
\hline & IP-T & 90 & $40.9 \%$ & $59.1 \%$ \\
\hline & LQS & 188 & $85.5 \%$ & $14.6 \%$ \\
\hline & GSM & 179 & $81.4 \%$ & $18.6 \%$ \\
\hline \multirow{4}{*}{ Uniform } & PCM & 132 & $60.0 \%$ & $40.0 \%$ \\
\hline & IP-T & 138 & $62.7 \%$ & $37.3 \%$ \\
\hline & LQS & 192 & $87.3 \%$ & $12.7 \%$ \\
\hline & GSM & 181 & $82.3 \%$ & $17.7 \%$ \\
\hline \multirow{4}{*}{ Random } & PCM & 26 & $11.8 \%$ & $88.2 \%$ \\
\hline & IP-T & 18 & $8.18 \%$ & $91.8 \%$ \\
\hline & LQS & 46 & $20.9 \%$ & $79.1 \%$ \\
\hline & GSM & 60 & $27.3 \%$ & $72.7 \%$ \\
\hline \multirow{4}{*}{$\begin{array}{c}\text { Random } \\
\text { All }\end{array}$} & PCM & 15 & $6.82 \%$ & $93.2 \%$ \\
\hline & IP-T & 8 & $3.64 \%$ & $96.4 \%$ \\
\hline & LQS & 17 & $7.73 \%$ & $92.3 \%$ \\
\hline & GSM & 9 & $4.11 \%$ & $95.9 \%$ \\
\hline
\end{tabular}




\section{A. SIP Signalling Evaluation:}

Fig. 4 shows the average calls setup time for VoIP applications over different mobility models and voice codecs. The Static and Uniform model have the best performance over all voice codecs (between 0.2 to 0.35 seconds), the Random model has a very high delays (between 4 to 8 seconds), and the Random All has the worst SIP call setup time. In general, VoIP Calls with LQS and GSM have the shortest SIP call setup time while PCM and IP-Telephony have long setup times over all mobility models.

The average setup time for VoIP calls is very unstable in Random mobility models of the effect of the TCP mechanism for SIP signaling and the hops number between MANET nodes. In Fig. 5, the average number of rejected calls over different mobility models is the lowest in the Uniform model compared with other mobility models. In addition, the LQS and GSM voice codecs have a low number of rejected calls. The number of rejected calls by even the SIP server or the Callee in Random and Random All mobility models are very high as a result of the route change, the high number of hops or the SIP connection initiation timeout.
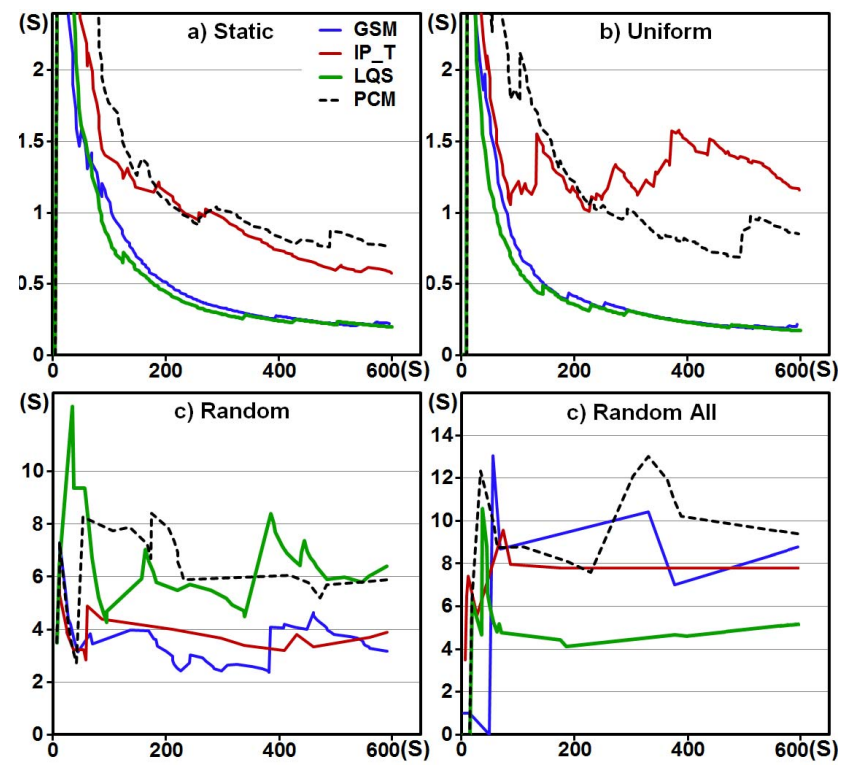

Figure 4. Average SIP Call Setup Time in Seconds.
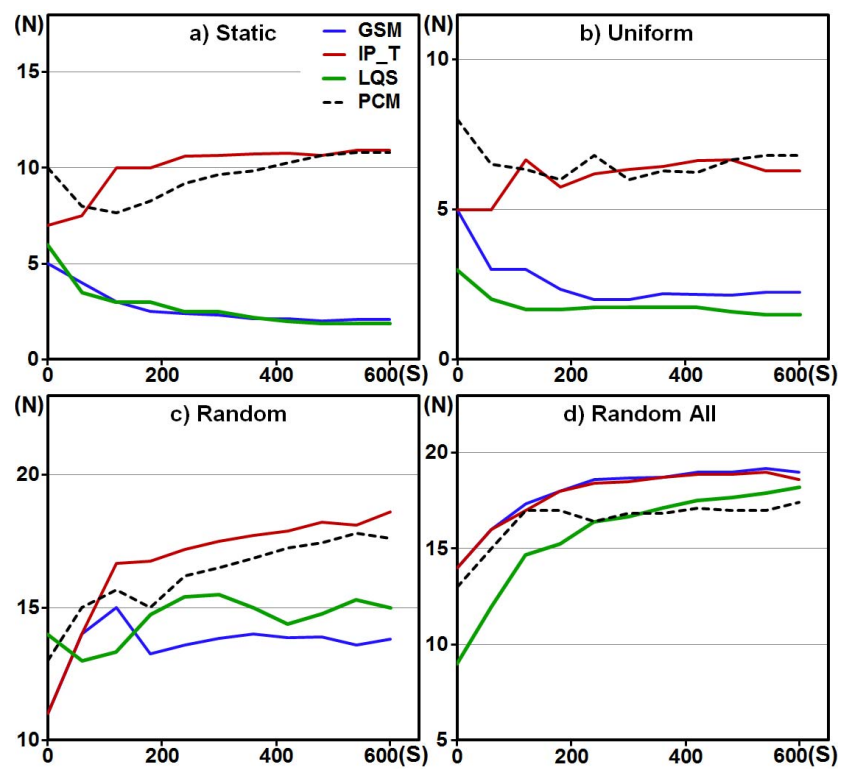

Figure 5. Average Number of Rejected SIP Calls.

\section{B. SIP Server Efficiency:}

The roll of the SIP server is very important to be investigated as its performance has direct impact on the SIP signaling and the VoIP QoS. The SIP server used to initiate, terminate and modify the SIP calls. The more number of calls the SIP server can support, the better performance can provide. To check the SIP server performance, a sample of VoIP calls had been examined for the performance of the SIP server. In Fig. 6, the average VoIP calls duration for the initiated and the terminated calls through the SIP server for calls with duration of 10 Seconds between node 22 and node 3 is about 11 seconds for LQS and GSM in both Static and Uniform models.
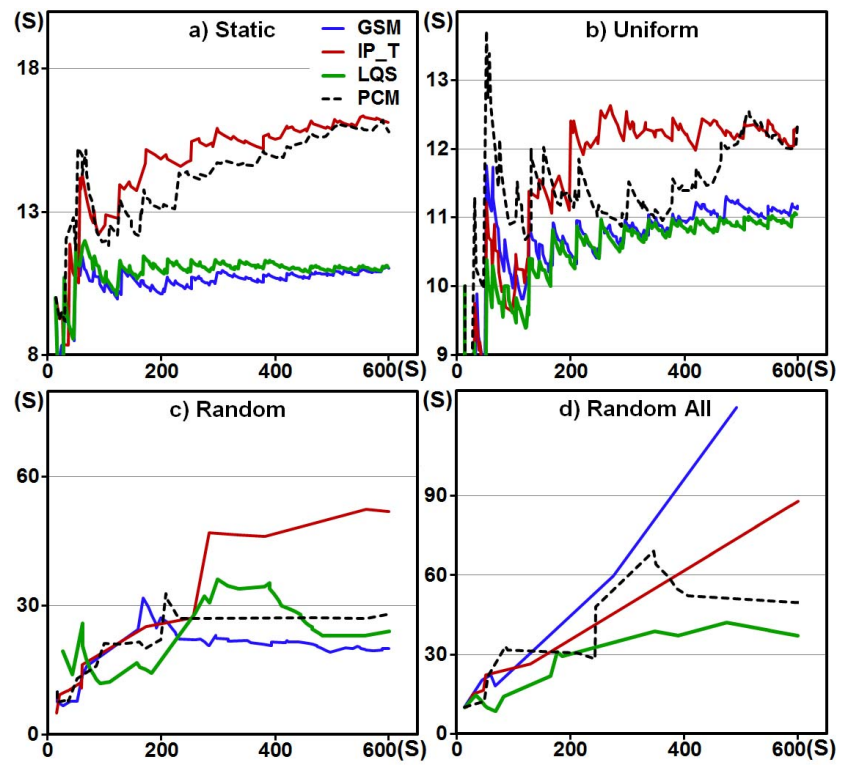

Figure 6. Average total time for VoIP Calls with 10 Seconds duration between node 22 and node 3 . 
This result shows that SIP server has good performance in this case. However, in the Random model the calls duration average is around 30 second which means there is more than 20 seconds delay in the SIP calls initiation/termination signaling for the limited number of successful VoIP calls. The signaling delays mostly happen as a result of the connectivity lost between the SIP server, and the Caller or the Callee. The retransmission mechanism of the SIP signaling on the SIP sever the callers sides have a direct impact on the SIP calls initiation/termination and the SIP performance. On the other hand, the PCM and the IPTelephony have higher delays for the calls with 10 Seconds duration over all types of mobility models as a result of the high bit rate and bandwidth consumption it has.

\section{Voice Signalling QoS:}

Fig. 7 shows the average delays for the RTP/UDP signals which represent the End-to-End delays for the voice packets. The voice packets are transferred over the OLSR MANET where the RTP delays affect the QoS of the VoIP calls. The PCM and the IP-Telephony has the highest RTP delays over all mobility models as both are generating high number of voice packets while LQS and GSM shown good performance over all mobility models. However, the RTP delay in Random models is very high with all voice codecs because both Random model have limited voice traffic. Moreover, the results shown that the RTP delays affects the other voice QoS parameters such as Jitter and Packets Delay Variation.
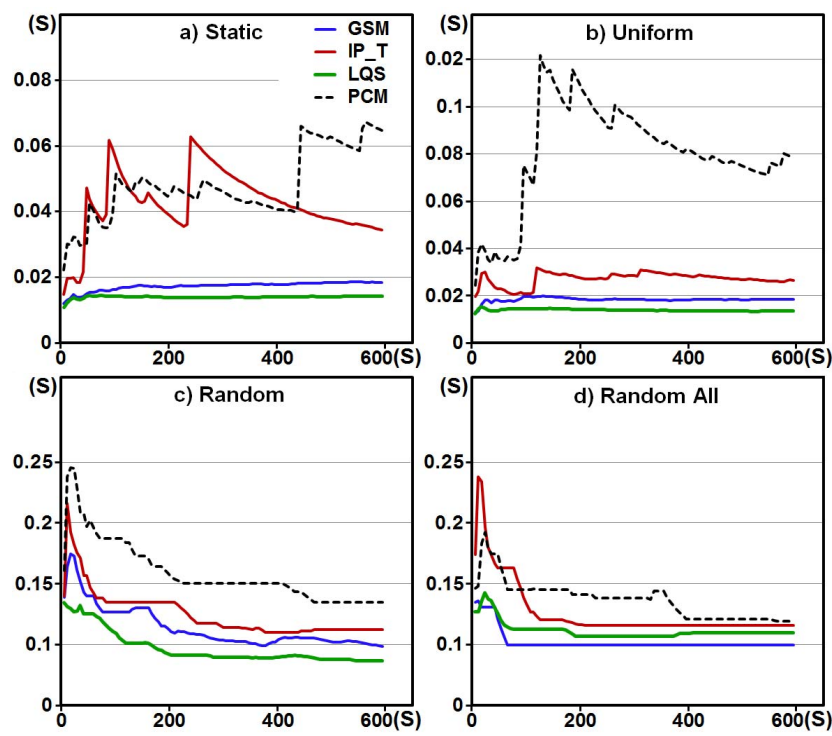

Figure 7. Average Voice Delay for RTP Signals.

Fig. 8 shows the average voice traffic sent from the Caller to the Callee in Bytes. The PCM has the largest amount of data for almost all mobility models then come the GSM. The amount of voice traffic reflects the ability of the Caller to call the Callee for that the sent traffic in the Static and
Uniform model is higher than Random models. In the Random models we find that the amounts of the data are high at the beginning of the simulation, but it falls down later. This is because of the initial location and the reachability of the MANET nodes at the beginning of the simulation time. The average number of received voice traffic in the receiver side is shown in Fig. 9. Because of the packet drops for some of the voice traffic between the Caller and the Callee, the amount of received traffic is lower than the sent traffic. By comparing the sent and the received traffic ratio, LQS has the smallest ratio of packets drop for voice traffic, then GSM while PCM and IP-Telephony have a large ratio of packets drop.
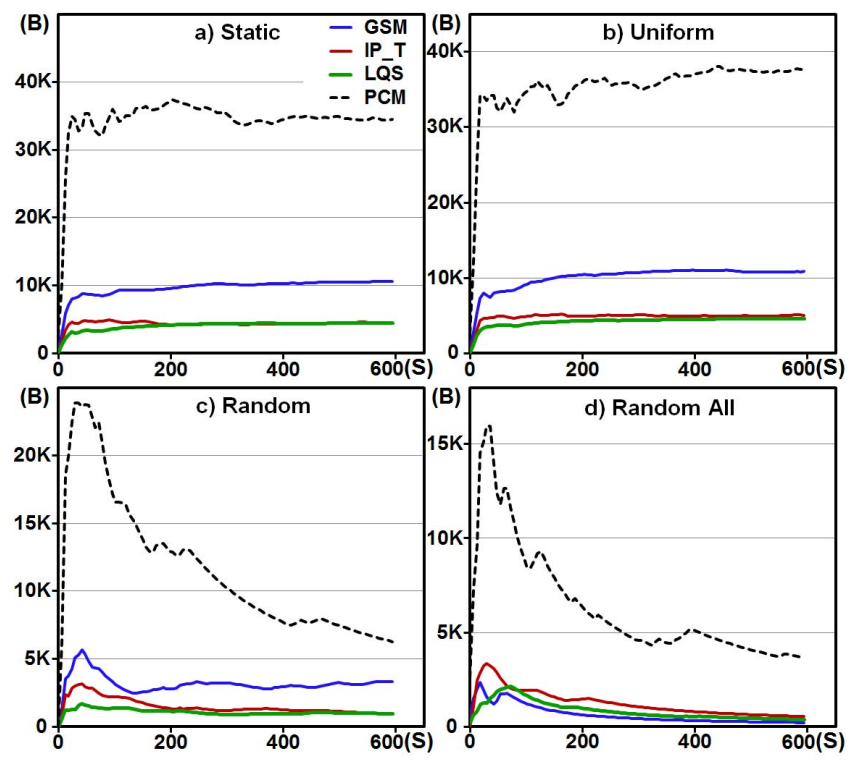

Figure 8. Average Voice Traffic Sent
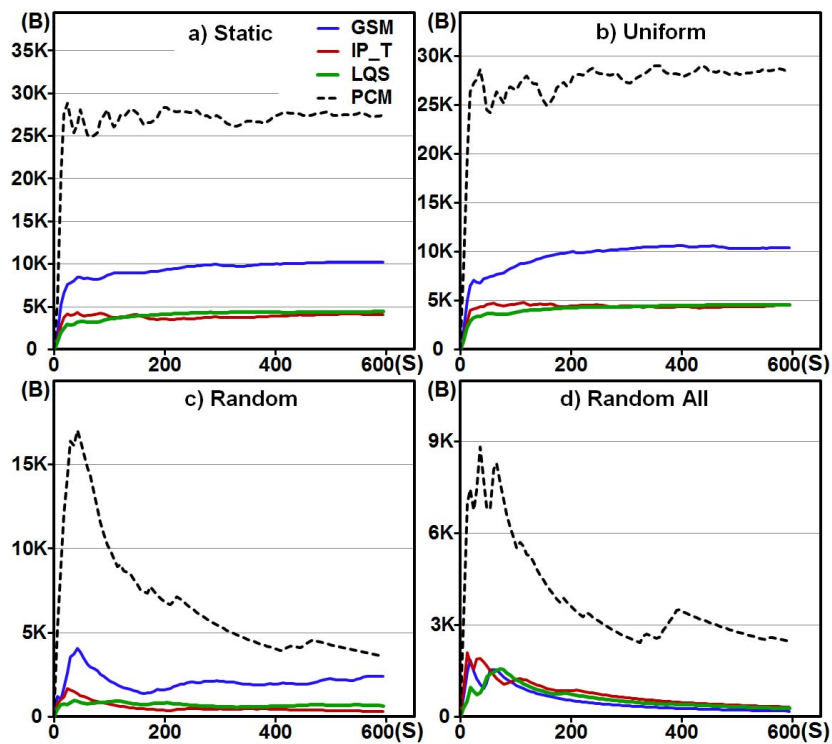

Figure 9. Average Voice Traffic Received 


\section{OLSR Performance:}

Both SIP and voice signaling performance are depending on OLSR routing algorithms as well as the calculation's performance of the routing tables. Fig. 10 shows the average calculation number of Multipoint Relay (MPR) selection for OLSR routing protocol. The MPR recalculation in OLSR happens whenever neighborhood change is detected on a node. The recalculation number of MPR depends on the type of the mobility model. The Static mobility model has a few number of MPR calculations while was increased in the Uniform models. The MPR calculation numbers for Radom models are almost triple the calculation numbers of the previous two models. The LQS and the GSM have a low number of MRP calculations except with Random mobility where the calculations are increased as the routs are intensively change.
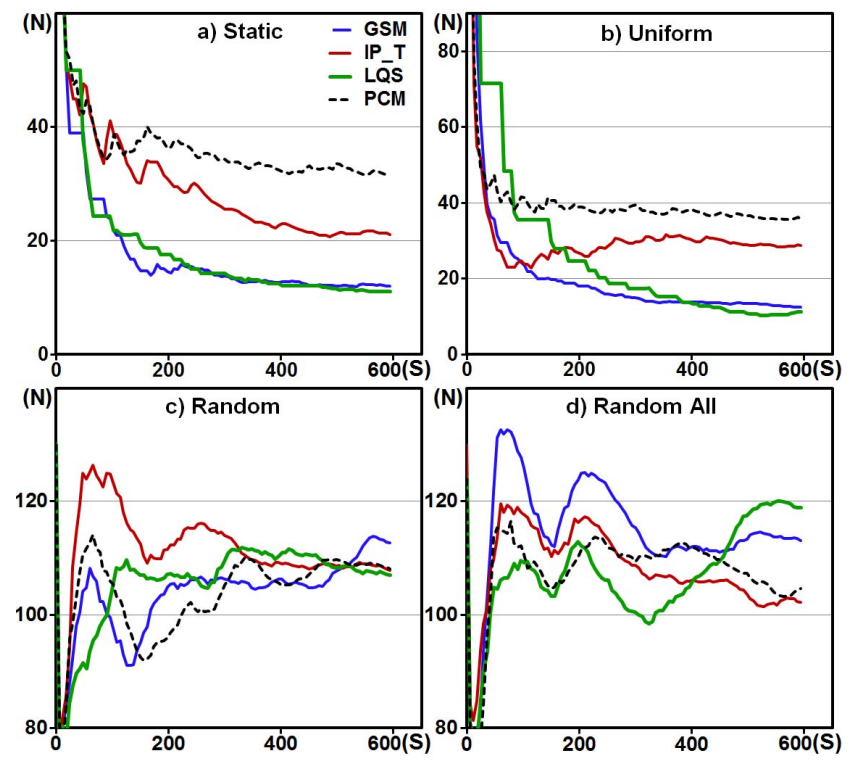

Figure 10. Average number of MPR calculations.

Fig. 11 shows the average calculation number of the OLSR route table calculations during the simulation. In OLSR, the re-calculations of the route table occur whenever a neighborhood or a topology change is detected on a node. The average calculation number for the routing table is high for PCM and IP-Telephony which is normal as the number of the voice packets for the both voice codecs are higher than LQS and GSM. The route calculations consume time and make more delays over the VoIP applications. However, the more number of calculations for mobile nodes means that real time applications could have better connectivity performance. There are no significant differences between the average number of the route table calculations for PCM and IP-Telephony over all mobility models while the LQS and the GSM have considerable differences. The OLSR over LQS and GSM show an increased number in the route table calculations as the changes of the topology and locations are very high, and OLSR is actively updating the routing tables to provide the connectivity for VoIP applications. This means that OLSR performance is better when the ratio of the routing protocol had increased with the increase of both, nodes location and the network topologies. For Random mobility models, GSM has the highest number of calculations for the routing table regarding to the total number of GSM packets compared with other voice codecs.

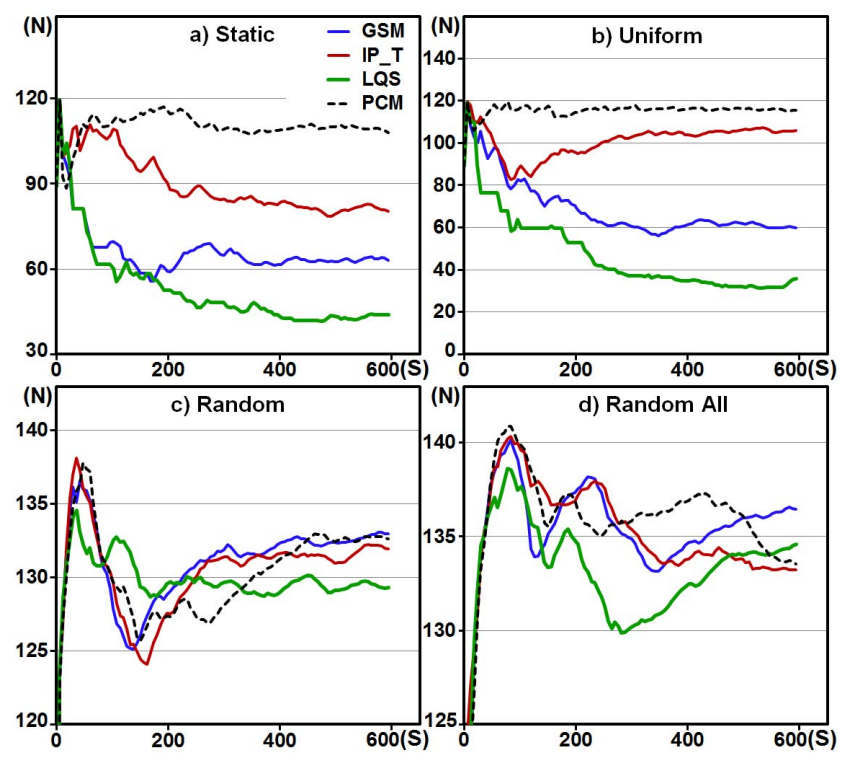

Figure 11. Average Route Tables Calculations.

\section{CONCLUSION AND FUTURE WORKS}

The evaluation efforts of this paper aim at highlighting the main performance matrices to study and improve the SIP based VoIP applications over OLSR MANET with different voice codecs and mobility models. The simulation results support the findings in [9] which states that OLSR behaves depending on the mobility model and the traffic type. In addition, OLSR shows good performance in terms of traffic load and transmission throughput. Static and Uniform mobility models have the best performance for all voice codecs while the Random model affected by the SIP server position and mobility.

In General, VoIP Calls with LQS and GSM have the shortest SIP call setup time while PCM and IP-Telephony have long setup times over all mobility models. Regarding voice QoS and SIP signaling, GSM has the best performance over different types of mobility models compared with other voice codecs as it has a good calculation performance for MPR and routing tables. Compared with LQS, GSM has a better voice quality even if LQS shown better results. PCM and IP-Telephony are inefficient for OLSR MANET realtime applications because of the long delays and the large amount of traffic. The TCP behavior affects SIP signaling and SIP delays could be enhanced by modifying the TCP timers. Furthermore, using other TCP versions may enhance the SIP signaling performance. The node capacity, the position and the mobility of the SIP serve are all affect the VoIP performance over OLSR MANET. 
The future works will focus on VoIP over Next Generation MANET. The current evaluation results based on IPv4 for OLSR MANET. The results highlight the performance trade off and the differences between both, IPv4 and IPv6, for SIP based VoIP applications. Furthermore, the SIP signaling enhancements and the SIP server performance will be considered for VoIP applications over IPv6 MANET.

\section{ACKNOWLEDGMENT}

The use of OPNET Modeler ${ }^{\circledR}$ version 17.1 in this research was facilitated through OPNET's University Program.

\section{REFERENCES}

[1] K. Pandey and A. Swaroop, "A Comprehensive Performance Analysis of Proactive, Reactive and Hybrid MANETs Routing Protocols, " IJCSI International Journal of Computer Science Issues, Vol. 8, No. 3, 2011.

[2] A. B. Johnston, "SIP Understanding the Session Initiation Protocol," third Edition, Artech House Publishers, 2009.

[3] S. Ganguly and S. Bhatnagar, "VoIP: Wireless, P2P and New Enterprise Voice Over IP," Chichester, England: Wiley, 2008. Print.

[4] H. kaur and E. J. Singh, "Performance comparison of OLSR, GRP and TORA using OPNET," International Journal of Advanced Research in Computer Science and Software Engineering, 2 (10), Oct-2012, pp. 1-6.

[5] P. Palta and S. Goyal, "Comparison of OLSR and TORA routing protocols using OPNET Modeler" in International Journal of Engineering Research and technology Vol. 1 Issue 5, July - 2012.

[6] M. S. Thompson, A. B. MacKenzie, and L. A. DaSilva, "A method of proactive MANET routing protocol evaluation applied to the OLSR protocol," Proceedings of the 6th ACM international workshop on Wireless network testbeds, experimental evaluation and characterization, pp. 27-34, 2011.

[7] I. Khan and A. Qayyum, "Performance evaluation of AODV and OLSR in highly fading vehicular ad hoc network environments," Multitopic Conference, 2009. INMIC 2009. IEEE 13th International, pp. $1-5,2009$

[8] S. Gandhi, N. Chaubey, P. Shah, and M. Sadhwani, "Performance evaluation of DSR, OLSR and ZRP protocols in MANETs," Computer Communication and Informatics (ICCCI), 2012 International Conference on, pp. 1-5, 2012.

[9] M. Amnai, Y. Fakhri, and J. Abouchabaka, "QoS Routing and Performance Evaluation for Mobile Ad Hoc Networks using OLSR Protocol," arXiv preprint arXiv: 1107.3656, 2011.

[10] P. Sondi and D. Gantsou, "Voice communication over mobile ad hoc networks: evaluation of a QoS extension of OLSR using OPNET," Asian Internet Engineering Conference, pp. 61-68, 2009.

[11] K. Jain, K. Somasundaram, B. Wang, J. Baras, and A. RoyChowdhury, "Study of OLSR for Real-time Media Streaming over 802.11 Wireless Network in Software Emulation Environment," 2010. 